\title{
The Role of Midkine in Breast Cancer
}

Walaa M. Hadida ${ }^{(1)}$, Dina A. Ali ${ }^{(1)}$, Ayman A. ELnemr ${ }^{(2)}$, Hanaa E. Nofal ${ }^{(1)}$

(1) Department of Clinical Pathology, (2) Department of Surgical Oncology

Faculty of Medicine - Tanta University

Corresponding author: Walaa M. Hadida; Mobile: 01069415772; Email: walaa.hadida@ gmail.com

\begin{abstract}
Background: Breast cancer (BC) is the most frequently diagnosed life-threatening cancer in women and the second leading cause of cancer death among women worldwide. The present conventional clinicopathological factors remain insufficient to evaluate the substantial prognosis of this disease. Midkine is a heparin-binding growth factor. It is upregulated in many types of cancer.

Aim of the work: The aim of this study was to through light on the role of serum midkine level in breast cancer patients and its relation to the conventional tumor marker CA 15-3.

Subject and method: This study was conducted on 40 newly diagnosed breast cancer patients (Group I) and 20 apparently healthy control subjects (Group II). Complete history, clinical examination, mammography and histopathological examination of breast cancer with immunohistochemical study were performed. Serum midkine level was assessed by double antibody sandwich assay technique(ELISA) by using human midkine ELISA kit also CA 15-3 level was assessed by using an automatic immune assay analyzer (TOSOH AIA system).

Results: The present study revealed a statistically significant difference between breast cancer patients (Group I) and healthy control (Group II) regarding serum midkine level. Serum midkine level showed a statistically significant difference with stage of breast cancer, menopausal status and CA 15-3 serum level. There was no statistically significant difference regarding age, family history of breast cancer, parity, breastfeeding and history of oral contraceptive pills (OCPs) intake.
\end{abstract}

Conclusions: This study showed that midkine might be a diagnostic and prognostic biomarker for breast cancer.

Keywords: Breast cancer, Midkine, ELISA, Immunoassay, CA 15-3.

\section{INTRODUCTION}

Breast cancer (BC) is the most widely spread diagnosed cancer among women and the second principle cause of cancer mortality. Deaths due to BC generally occur due to the growth of distant metastases that cannot be detected with the existing treatments. Approximately $69 \%$ of women who are diagnosed with advanced-stage breast cancer die within 5 years ${ }^{(1)}$.

$\mathrm{BC}$ is a quite complex and heterogeneous in its development, progress, and response to treatment; therefore, researchers are encouraged to identify novel biomarkers for the optimization of breast carcinoma management ${ }^{(2)}$.

The key to clinical diagnosis and treatment of breast cancer is early diagnosis of BC. Accurate determination of the clinical condition, and the detection of related molecules in tumor tissue can provide accurate evidence for the judgment of pathological stage and the degree of tumor malignancy. Tumor tissue obtained through biopsy, on the other hand, may cause a certain degree of trauma to patients ${ }^{(3)}$.

Growth factors contribute largely to tumor formation. They stimulate cell proliferation and regulate cell differentiation, migration, survival, and proliferation. Many studies reported that high levels of growth factors associated with malignant tumors and such factors play a role in tumor development, growth, invasion, angiogenesis and metastasis ${ }^{(3)}$.
Midkine (MK) is a multifunctional growth factor that can induce various effects in the targeted cells including nerve cells, neutrophils, macrophages, smooth muscle cells, fibroblasts, mammary gland cells and tumor cells. It plays a role in tumor development, growth, invasion, angiogenesis, and metastasis. For this reason, some researchers have focused on investigating the MK expression as one of the growth factors and on the relationship of this expression with cancer types and its serum values ${ }^{(\mathbf{1})}$.

\section{SUBJECTS AND METHODS Subjects:}

This study was conducted on 40 newly diagnosed breast cancer patients who were recruited from the Surgical Oncology Outpatient Clinic in Tanta University Hospitals. In addition, 20 apparently healthy subjects serving as healthy control matched by age, and geographic origin with the patients group. Subjects included in this study were classified into the following groups:

\section{A. Group I: breast cancer patients $(\mathrm{BC})=$ Cases $(n=40)$ :}

This group included 40 newly diagnosed breast cancer patients classified according to findings into stage I, II, III and IV. Their age ranged from 40 to 70 years old with a mean of $54.50 \pm 9.5$ (Mean \pm SD). 


\section{B. Group II: Healthy Control Group = Control $(\mathbf{n}=20)$ :}

This group included 20 apparently healthy control Subjects. Their age ranged from 42 to 70 years old with a mean of $53.90 \pm 10.27$ years.

The study was approved by the Ethical Committee of Tanta University, and a written informed consent was obtained from all participants.

\section{Ethical approval:}

The study was approved by the Ethics Board of Tanta and an informed written consent was taken from each participant in the study.

\section{Provision of privacy:}

Privacy of all data was guaranteed and there was a code number for every patient and included all her investigations.

\section{Inclusion criteria;}

This study was carried out on newly diagnosed patients admitted to the Surgical Oncology Outpatient Clinic depending on:

1- Presence of breast mass

2-Mammography

3-Histopathological examination, grading and staging were determined.

\section{Exclusion criteria;}

Breast cancer patients with another type of he analysis and statistical presentation of the current study was cancer, liver dysfunction, diabetes mellitus, rheumatoid arthritis, and other inflammatory diseases were excluded from this study.

\section{All patients were subjected to the following:}

1- Complete history taking including personal, reproductive and family history.

2- Clinical examination with particular attention to organomegaly, L.Ns enlargement and any other masses.

3- Routine laboratory investigations;

- Blood glucose level:

- Fasting blood glucose level.

- Postprandial blood glucose level.

- Liver function tests.

- Kidney function tests.

- CBC, ESR, LDH and CRP.

4- Tumor marker;

- CA15-3 serum level assessed by immunoenzymometric method $^{(4)}$.

5- Specific investigations;

- Measurement of serum midkine level by ELISA technique ${ }^{(4)}$.
Sample:

- A sex milliliter (6 ml) of venous blood were drown from every subject under complete aseptic technique and emptied in three tubes:

- $1 \mathrm{ml}$ blood was put into ethylene diamine tetra-acetic acid (EDTA) tube for complete blood picture assessment.

- $1.6 \mathrm{ml}$ blood was put into sodium citrate tube for ESR assessment.

- The remaining three $\mathrm{ml}$ blood were emptied into a serum separator tube (SST) then allowed to clot for 30 minutes before centrifugation for 15 minutes at approximately $1000 \times \mathrm{g}$. Serum of each sample was removed and divided into two parts one was used for assessment of routine laboratory investigations and CA15-3 and the other was stored at $-20^{\circ} \mathrm{C}$ till the time of assay of level of midkine. Frozen samples were allowed to thaw, then, brought to room temperature only before analysis. Hemolysed samples were discarded; repeated thawing and freezing were avoided.

\section{Method:}

Assessment of midkine level:

Serum midkine level was assessed by using the commercially available human midkine ELISA technique (double antibody sandwich technique) ${ }^{(4)}$.

\section{Statistics}

depending on the following tests using SPSS; mean standard deviation and Chi-Square. Student "t. test" to show the significance of the difference between two means. Linear Correlation Coefficient [r]. Receiver operating characteristic (ROC curve) where analysis was used to find out the overall predictivity of parameter in and to find out the best cut-off value with detection of the sensitivity and the specificity at this cut-off value. Oneway analysis of variance (ANOVA) to analyze the differences among group of means in a sample.

\section{RESULTS}

Table (1): Comparison between BC patients (Group I) and control (Group II) regarding age

\begin{tabular}{|l|c|c|}
\hline \multicolumn{1}{|c|}{ Age } & $\begin{array}{c}\text { patients (Group I) } \\
(\mathbf{n = 4 0 )}\end{array}$ & $\begin{array}{c}\text { ontrol (Group II) } \\
(\mathbf{n = 2 0})\end{array}$ \\
\hline Range & $40-70$ & $42-70$ \\
\hline $\begin{array}{l}\text { Mean } \pm \\
\text { S.D }\end{array}$ & $54.50 \pm 9.51$ & $53.90 \pm 10.27$ \\
\hline t.test & \multicolumn{2}{|c|}{0.031} \\
\hline p-value & \multicolumn{2}{|c|}{0.861} \\
\hline
\end{tabular}

* Significant $p$ value $<0.05$ 
Table (1) showed that there was no statistically significant difference between BC patients (Group I) and control (Group II) regarding age $(\mathrm{p}$-value $=0.861)$.

Table (2): Comparison between BC patients (Group I) and control (Group II) regarding family history of breast cancer

\begin{tabular}{|l|l|l|l|l|}
\hline $\begin{array}{l}\text { Family } \\
\text { History }\end{array}$ & \multicolumn{2}{|c|}{$\begin{array}{c}\text { BC patients } \\
\text { (Group I) } \\
(\mathbf{n = 4 0})\end{array}$} & $\begin{array}{c}\text { Control } \\
\text { (Group II) } \\
(\mathbf{n = 2 0})\end{array}$ \\
\hline & $\mathbf{N}$ & $\mathbf{\%}$ & $\mathbf{N}$ & $\%$ \\
\hline Positive & 23 & 57.5 & 2 & 10 \\
\hline Negative & 17 & 42.5 & 18 & 90 \\
\hline $\mathbf{X}^{\mathbf{2}}$ & \multicolumn{3}{|c|}{12.382} \\
\hline p-value & \multicolumn{3}{|c|}{$0.001 *$} \\
\hline
\end{tabular}

* Significant $p$ value $<0.05$

Table (2) showed that there was a statistically significant difference between BC patients (Group I) and control (Group II) regarding family history of breast cancer $\left(\mathrm{p}\right.$-value $\left.=0.001^{*}\right)$.

Table (3): Comparison between BC patients (Group I) and control (Group II) regarding parity

\begin{tabular}{|c|r|r|r|r|}
\hline Parity & $\begin{array}{c}\text { BC patients } \\
\text { (Group I) } \\
(\mathbf{n = 4 0 )}\end{array}$ & $\begin{array}{l}\text { Control } \\
\text { (Group II) } \\
(\mathbf{n = 2 0})\end{array}$ \\
\hline & $\mathbf{N}$ & $\mathbf{\%}$ & $\mathbf{N}$ & $\%$ \\
\hline Nulliparous & 25 & 62.5 & 2 & 10 \\
\hline $\begin{array}{l}\text { One or more } \\
\text { (multiparous) }\end{array}$ & 15 & 37.5 & 18 & 90 \\
\hline $\mathbf{X}^{\mathbf{2}}$ & \multicolumn{3}{|c|}{14.851} \\
\hline p-value & \multicolumn{3}{|c|}{$0.001^{*}$} \\
\hline
\end{tabular}

* Significant $p$ value $<0.05$

Table (3) showed that there was a statistically significant difference between BC patients (Group I) and control (Group II) regarding parity $\left(\mathrm{p}\right.$-value $\left.=0.001^{*}\right)$.

Table (4): Comparison between BC patients (Group I) and Control (Group II) regarding breastfeeding

\begin{tabular}{|c|c|c|c|c|}
\hline Breastfeeding & \multicolumn{2}{|c|}{$\begin{array}{c}\text { BC patients } \\
\text { (Group I) } \\
(\mathbf{n = 4 0 )}\end{array}$} & $\begin{array}{c}\text { Control } \\
\text { (Group II) } \\
(\mathbf{n = 2 0})\end{array}$ \\
\hline & $\mathbf{N}$ & $\mathbf{\%}$ & $\mathbf{N}$ & \% \\
\hline Positive & 17 & 42.5 & 16 & 80 \\
\hline Negative & 23 & 57.5 & 4 & 20 \\
\hline $\mathbf{X}^{\mathbf{2}}$ & \multicolumn{4}{|c|}{7.582} \\
\hline p-value & \multicolumn{4}{|c|}{$0.006^{*}$} \\
\hline
\end{tabular}

* Significant $p$ value $<0.05$

Table (4) showed that there was a statistically significant difference between BC patients (Group I) and control (Group II) regarding breastfeeding ( $\mathrm{p}$-value = $0.006 *)$.

Table (5): Comparison between BC patients (Group I) and control (Group II) regarding history of OCPs intake

\begin{tabular}{|c|c|c|c|c|}
\hline \multirow[t]{2}{*}{$\begin{array}{c}\text { History } \\
\text { of OCPs }\end{array}$} & \multicolumn{2}{|c|}{$\begin{array}{c}\text { BC patients } \\
(\text { Group I) } \\
(n=40)\end{array}$} & \multicolumn{2}{|c|}{$\begin{array}{c}\text { Control } \\
(\text { Group II) } \\
(\mathbf{n}=\mathbf{2 0})\end{array}$} \\
\hline & $\mathbf{N}$ & $\%$ & $\overline{\mathbf{N}}$ & $\%$ \\
\hline Positive & 27 & 67.5 & 6 & 30 \\
\hline Negative & 13 & 32.5 & 14 & 70 \\
\hline$X^{2}$ & \multicolumn{4}{|c|}{7.583} \\
\hline p-value & \multicolumn{4}{|c|}{$0.006^{*}$} \\
\hline
\end{tabular}

* Significant $p$ value $<0.05$

Table (5) showed that there was a statistically significant difference between BC patients (Group I) and control (Group II) regarding history of OCPs intake (pvalue $\left.=0.006^{*}\right)$.

Table (6): Comparison between BC patients (Group I) and control (Group II) regarding menopausal status

\begin{tabular}{|c|l|r|r|r|}
\hline $\begin{array}{r}\text { Menopausal } \\
\text { Status }\end{array}$ & \multicolumn{2}{|c|}{$\begin{array}{c}\text { BC patients } \\
\text { (Group I) } \\
(\mathbf{n = 4 0 )}\end{array}$} & $\begin{array}{c}\text { Control } \\
\text { (Group II) } \\
(\mathbf{n = 2 0})\end{array}$ \\
\hline & N & \% & $\mathbf{N}$ & $\%$ \\
\hline $\begin{array}{c}\text { Pre- } \\
\text { menopausal }\end{array}$ & 12 & 30 & 12 & 60 \\
\hline $\begin{array}{c}\text { Post- } \\
\text { menopausal }\end{array}$ & 28 & 70 & 8 & 40 \\
\hline $\mathbf{X}^{2}$ & \multicolumn{4}{|c|}{5.002} \\
\hline p-value & \multicolumn{4}{|c|}{$0.025^{*}$} \\
\hline
\end{tabular}

* Significant $p$ value $<0.05$

Table (6) showed that there was a statistically significant difference between BC patients (Group I) and control (Group II) regarding menopausal status (p-value $\left.=0.025^{*}\right)$.

Table (7): Comparison between BC patients (Group I) and control (Group II) regarding serum midkine level $(\mathrm{pg} / \mathrm{ml})$

\begin{tabular}{|r|c|c|}
\hline Midkine & $\begin{array}{c}\text { BC patients } \\
(\text { Group I) } \\
(\mathbf{n = 4 0})\end{array}$ & $\begin{array}{c}\text { Control } \\
(\text { Group II) } \\
(\mathbf{n = 2 0})\end{array}$ \\
\hline Range & $136.81-471.50$ & $64.80-149.8$ \\
\hline Mean \pm S.D & $251.68 \pm 92.90$ & $118.83 \pm 25.05$ \\
\hline t.test & \multicolumn{2}{|c|}{19.805} \\
\hline p-value & \multicolumn{2}{|c|}{$0.001 *$} \\
\hline
\end{tabular}

*Significant $p$ value $<0.05$

Table (7) showed that there was a statistically significant difference between BC patients (Group I) and 
control (Group II) regarding serum midkine level ( $\mathrm{p}$ value $\left.=0.001^{*}\right)$.

Table (8): Comparison between BC patients (Group I) and control (Group II) regarding serum CA 15-3 level $(\mathrm{U} / \mathrm{ml})$

\begin{tabular}{|c|c|c|}
\hline CA 15-3 & $\begin{array}{c}\text { patients (Group I) } \\
(\mathbf{n = 4 0 )}\end{array}$ & $\begin{array}{r}\text { Control(Group II) } \\
(\mathbf{n = 2 0})\end{array}$ \\
\hline Range & $65.7-117.3$ & $15-30$ \\
\hline ean \pm S.D & $88.87 \pm 14.50$ & $22.10 \pm 4.69$ \\
\hline t.test & \multicolumn{2}{|c|}{140.184} \\
\hline p-value & \multicolumn{2}{|c|}{$0.001^{*}$} \\
\hline
\end{tabular}

* Significant $p$ value $<0.05$

Table (8) showed that there was a statistically significant difference between BC patients (Group I) and control (Group II) regarding serum CA 15-3 level (pvalue $\left.=0.001^{*}\right)$.

Table (9): Correlation between serum midkine level and serum CA 15-3 level in BC patients group

\begin{tabular}{|l|l|l|}
\hline \multirow{2}{*}{ CA 15-3 } & \multicolumn{2}{|c|}{ Midkine } \\
\cline { 2 - 3 } & r. & P \\
\hline & 0.828 & $0.001^{*}$ \\
\hline
\end{tabular}

*Significant p value $<0.05$

Table (9) showed that there was a statistically significant positive correlation between serum midkine level and serum CA15-3 level in BC patients group (pvalue $=0.001 *)$.

Table (10): Serum midkine level in different stages of breast cancer and control

\begin{tabular}{|c|c|r|r|r|r|}
\hline \multirow{2}{*}{ Midkine } & \multirow{2}{*}{$\begin{array}{c}\text { Nontrol } \\
(\mathbf{n}=20)\end{array}$} & $\begin{array}{c}\text { Stage I } \\
(\mathbf{n}=10)\end{array}$ & $\begin{array}{r}\text { Stage II } \\
(\mathbf{n}=10)\end{array}$ & $\begin{array}{r}\text { Stage III } \\
(\mathbf{n}=10)\end{array}$ & $\begin{array}{r}\text { Stage IV } \\
(\mathbf{n}=10)\end{array}$ \\
\hline Range & $64.80-$ & $136.81-$ & $178.42-$ & $233.66-$ & $324.67-$ \\
& 133.08 & 174.15 & 232.03 & 301.91 & 471.91 \\
\hline Mean & 114.83 & 153.36 & $200.88 \pm$ & $267.30 \pm$ & $385.19 \pm$ \\
\pm S.D & \pm 21.21 & \pm 14.17 & 19.05 & 22.81 & 51.25 \\
\hline F.test & \multicolumn{5}{|c|}{108.959} \\
\hline p-value & \multicolumn{5}{|c|}{$0.001^{*}$} \\
\hline I \& II & I \& III & $\mathbf{1} \&$ IV & II \& III & II \& IV & III \& IV \\
\hline $0.003^{*}$ & $0.001^{*}$ & $0.001^{*}$ & $0.001^{*}$ & $0.001^{*}$ & $0.001^{*}$ \\
\hline
\end{tabular}

*Significant $p$ value $<0.05$

Table (10) showed that there was a statistically significant difference between control and stage I of breast cancer regarding serum midkine level $(\mathrm{p}$-value $=$ $0.001 *)$.

Also, There was a statistically significant difference among different stages of breast cancer regarding serum midkine level where P1 (I \& II) $=0.003$
*, while P2 (I \& III), P3 (I \& IV), P4 (II \& III), P5 (II \& IV) and P6 (III \& IV) $=0.001 *$.

Table (11): Correlation between serum midkine level and age in $\mathrm{BC}$ patients group

\begin{tabular}{|c|c|c|}
\hline \multirow{2}{*}{ Age } & \multicolumn{2}{|c|}{ Midkine } \\
\cline { 2 - 3 } & r. & p-value \\
\hline & 0.214 & 0.184 \\
\hline
\end{tabular}

* Significant $p$ value $<0.05$

Table (11) showed that there was no statistically significant differences between serum midkine level and age in $\mathrm{BC}$ patients group ( $\mathrm{p}$-value $=0.184$, correlation $(\mathrm{r})$ $=0.214$ ).

Table (12): Relation between serum midkine level and menopausal status in BC patients (Group I)

\begin{tabular}{|c|c|c|}
\hline \multirow{2}{*}{ Midkine } & \multicolumn{2}{|c|}{ Menopausal status } \\
\cline { 2 - 3 } & $\begin{array}{c}\text { Pre-menopausal } \\
(\mathrm{n}=12)\end{array}$ & $\begin{array}{c}\text { Post-menopausal } \\
(\mathrm{n}=28)\end{array}$ \\
\hline Range & $136.81-460.00$ & $136.81-471.50$ \\
\hline Mean \pm S.D & $186.79 \pm 75.50$ & $294.94 \pm 77.68$ \\
\hline T test & \multicolumn{2}{|c|}{19.025} \\
\hline P value & \multicolumn{2}{|c|}{$0.001 *$} \\
\hline
\end{tabular}

*Significant $p$ value $<0.05$

Table (12) showed a statistically significant relation between serum midkine level and menopausal status in BC patients (Group I) (p-value $=0.001 *)$.

Table (13): Sensitivity and specificity of serum midkine in breast cancer

\begin{tabular}{|c|c|}
\hline & Midkine \\
\hline AUC & $0.965 \mathrm{pg} / \mathrm{ml}$ \\
\hline Cutoff & $>140 \mathrm{pg} / \mathrm{ml}$ \\
\hline Sensitivity \% & $95 \%$ \\
\hline Specificity \% & $97 \%$ \\
\hline PPV & $90 \%$ \\
\hline NPV & $82 \%$ \\
\hline Accuracy & $94 \%$ \\
\hline P- value & $0.001 *$ \\
\hline
\end{tabular}

* Significant $p$ value $<0.05$

Table (13) \& figure (1) showed receiver operating curve for serum midkine showing the optimum diagnostic cutoff $>140 \mathrm{pg} / \mathrm{ml}$, with sensitivity 95\%, specificity 97\%, PPV 90\%, NPV $82 \%$ and accuracy $94 \%$ (p-value $=0.001 *)$. 


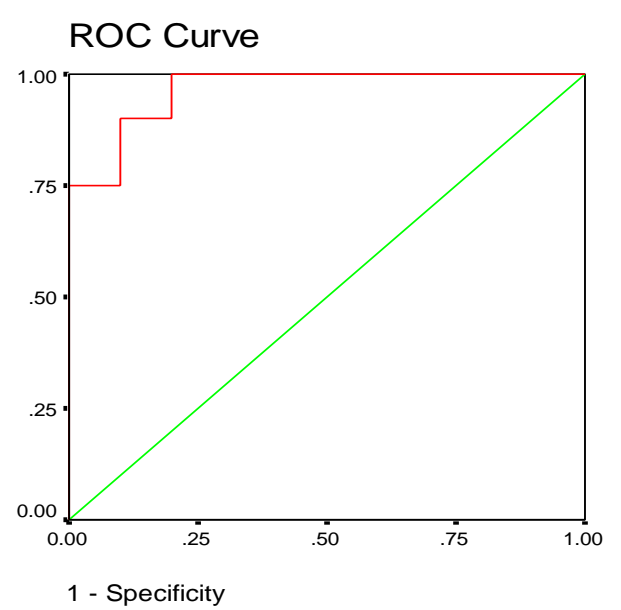

Figure (1): ROC curve of serum midkine in breast

\section{DISCUSSION}

cancer

Breast cancer is the most common malignancy among women worldwide and the second most common cause of cancer mortality. It is a clinically heterogeneous and complex disease. Several risk factors for breast cancer have been established as age, inherited genetic mutations, such as in the BRCA1 and BRCA2 genes, and a personal or family history of BC. Other risk factors include nulliparous females, greater height, benign breast disease, early menarche, late menopause (after age 54 years old), ionizing-radiation exposure, post-menopausal obesity, physical inactivity, and alcohol intake. Breastfeeding, moderate/vigorous physical activity, and maintaining a healthy body weight decrease breast cancer risk. High breast tissue density and high circulating levels of estrogens have also been associated with higher breast cancer incidence ${ }^{(5)}$.

Breast cancer cells have strong proliferation and anti-apoptosis ability. In the development process, cancer cells display infiltrative growth and a variety of cytokines are associated with these biological behavior of tumor cells during the development process of $\mathrm{BC}$, local angiogenesis as well as cancer cell invasion are important pathological links of the malignancy ${ }^{(3)}$.

Midkine, a cytokine, which is a member of heparin-binding growth factors family was a highly expressed factor during the early differentiation stage in embryogenesis and was weak or undetectable in normal adult tissues. MK has various biological activities such as proliferation, migration, anti-apoptosis, angiogenesis, invasion, and metastasis ${ }^{\left({ }^{6}\right)}$.

The results of this work revealed a significant relationship between family history of breast cancer and risk of BC development. In agreement with this result, Haber et al ${ }^{(7)}$ and Macías-Gómez et al. ${ }^{(8)}$ observed an increased breast cancer risk in females who had a positive family history of BC. This was confirmed also by Brewer et al. ${ }^{(9)}$ who showed that the role of family history in breast cancer incidence had been well established where females with a family history of breast carcinoma, especially in a first-degree relatives are at increased risk. The risk is higher with more than one affected first-degree relative compared to females without a family history.

In addition, in the present study, there was a significant relationship between parity and breast cancer risk. Jiang et al. ${ }^{(10)}$ proved that high parity had been associated with low breast cancer risk, while null parity was associated with an overall increased risk of BC.

The present study showed that there was a significant relationship between non-breastfeeding and breast cancer risk. EIShmay ${ }^{(11)}$ and Khincha et al. ${ }^{(12)}$ revealed that breastfeeding for any duration reduced risk of breast cancer. The risk was significantly reduced with breastfeeding for at least 6 months.

Concerning history of OCPs intake the present study demonstrated a significant relationship with breast cancer development. This was supported by the results of a study done by Soroush et al. ${ }^{(13)}$ who showed that OCPs was associated with increased risk of the breast cancer so that the breast cancer risk in the women who used OCPs was 1.5 greater than that in the women who didn't use pills. However, they also found that the longer the duration of OCPs consumption the greater the risk of BC.

The results of this study showed that breast cancer was higher in post-menopausal females than premenopausal ones. Fauci et al. (14) reported that menopause does not cause cancer development, but the risk of developing breast carcinoma increases as female ages due to longer exposure to estrogen. In agreement with that, Kamińska et al. ${ }^{(15)}$ explained that BC was most frequently found in women around menopause. It was significantly less frequently found in women below 45 years of age.

As regards, the result of midkine, serum midkine level was higher in BC patients than in healthy control group. In agreement with this result Ibusuki et al. ${ }^{(4)}$ revealed that serum midkine level was higher in BC patients than in controls. Sorkun et al. ${ }^{(\mathbf{1})}$ showed that the serum level of midkine was elevated even at early stages of BC. Moreover, it decreased after the tumor excision. This was confirmed also by Jing et al. ${ }^{\left({ }^{(6)}\right.}$ who reported that $\mathrm{MK}$ is one of the most aberrant secreted proteins that increased in the early stages of numerous tumors. It was revealed that serum MK level was high in precancerous lesions and the early stage of carcinoma development.

Zhang ${ }^{(3)}$ and Li et al. ${ }^{(16)}$ explained the role of midkine in breast cancer where they demonstrated that 
serum midkine level in $\mathrm{BC}$ patients abnormally rises where high expression of MK can induce breast cancer cell proliferation, inhibit breast cancer cell apoptosis and promote angiogenesis and cell invasion. MK overexpression had been reported in various cancer types for malignancies in all major organs and tissue types, ranging from the most common cancers to some of the rarest. Overexpression of both the MK gene and the MK protein within the tumor is a typical feature of cancer ${ }^{(\mathbf{1 7})}$. High expression of MK in cancer tissues was observed in many cancers and is associated with poor prognosis. High MK blood levels are also detected in a variety of cancers and are a predictive factor in various tumors ${ }^{(18)}$. In addition, $\mathbf{L i}$ et al. ${ }^{(16)}$ reported that MK had several mechanisms in breast cancer development process. These include, induction of cancer cell proliferation, cell survival, anti-apoptosis, tumorigenesis, and epithelialmesenchymal transition (EMT). Serum MK concentration is expected to increase with respect to the presence of MK-expressing tumors.

Several studies showed that these functions of MK were derived from activation of the mitogenactivated protein kinase (MAPK) and phosphatidyl inositol 3-kinase (PI3K) /Akt pathways by the MK receptor anaplastic lymphoma kinase (ALK), activation of the extracellular signal-regulated kinase 1/2 (ERK1/2), and PI3K pathways via protein tyrosine phosphatase $\zeta$ $(\mathrm{PTP} \zeta)$ (another MK receptor), activation of the Janus tyrosine kinase (JAK) /signal transducer, and activator of transcription (STAT) pathway, as well as activation of notch signaling ${ }^{(\mathbf{1 9})}$.

The result of this study showed that there was a significant relation between serum CA 15-3 level and breast cancer as serum CA 15-3 level was higher in BC patients than in healthy control group. A similar results was obtained by Nisman $\boldsymbol{e t}$ al. ${ }^{(20)}$ who revealed that patients with breast cancer showed higher CA 15-3 serum concentrations than those of the control group so estimations of CA 15-3 in the diagnosis of BC patients are useful.

The present study revealed that there was a significant correlation between serum midkine level and serum CA15-3 level in BC patients group. These results were consistent with Ibusuki et al. ${ }^{(4)}$ who revealed that there was a positive correlation between serum midkine level and serum CA15-3 level in BC patients.

The present study revealed a significant difference between control and stage I of breast cancer regarding serum midkine level, as serum MK levels significantly increased in stage I of BC patients as compared to control group. Ibusuki et al. ${ }^{(4)}$ and Zhang et al. ${ }^{(21)}$ found that serum midkine level in healthy control subjects was less than those in stage I of BC patients.
Also a significant difference was demonstrated between different stages of breast cancer and serum midkine level in BC patients as serum MK level in stage II was higher than stage I BC, stage III was higher than stage II and stage IV was higher than stage III. In agreement with this finding, Li et al. ${ }^{(16)}$ demonstrated that higher expression of midkine in breast cancer tissues was correlated with breast cancer progression including advanced clinical stage. In addition, Zhang ${ }^{(3)}$ reported that MK expression in breast tissue and serum MK content of breast cancer patients was significantly related with different clinical stages of breast cancer.

In contrast with this result, Ibusuki et al. ${ }^{(4)}$ and Yamashita et al. ${ }^{(22)}$ revealed that serum midkine level had no relation to clinical stage of breast cancer.

This study revealed that there was no relation between age and serum midkine concentrations among BC patients. This result was in agreement with Sorkun et al. ${ }^{(1)}$, Ibusuki et al. ${ }^{(4)}$ and Li et al. ${ }^{\left({ }^{(1)}\right)}$ who revealed that there was no relation between age and serum midkine level. On the other hand, Yamashita et al. ${ }^{(22)}$ reported that serum MK levels influenced by age of patient.

In the current study, there was a significant relation between menopausal status and serum midkine level in BC patients group. Ibusuki et al. ${ }^{(4)}$ and Jono and Ando ${ }^{(23)}$ reported that serum MK levels were significantly correlated with menopausal status as it was found that serum MK levels were significantly higher in post-menopausal patients than premenopausal in breast cancer patients.

The results of this study revealed that serum midkine had $95 \%$ sensitivity and $97 \%$ specificity at cutoff $>140 \mathrm{pg} / \mathrm{ml}$ for diagnosis of breast cancer. These results are close to those reported by Ibusuki et al., (4) who found that serum midkine had $80 \%$ sensitivity and $83 \%$ specificity at cut-off $0.654 \mathrm{pg} / \mathrm{ml}$ for early detection of breast cancer.

Moreover, Breast cancer diagnosis that based on MK levels was more specific than those based on three conventional markers including CEA, carbohydrate antigen 15-3 (CA15-3) and Nation Cancer CenterStomach-439 (NCC-ST-439) ${ }^{(23)}$.

\section{CONCLUSION}

From this study, it was concluded that:

MK levels were significantly elevated in BC patients compared with healthy control samples matched in age and geographical distribution. Moreover, serum midkine level positively correlated with clinical staging of breast cancer, menopausal status and serum CA 15-3 in $\mathrm{BC}$ patients. The high sensitivity of MK suggest that it may be a novel marker for early breast cancer detection. In addition, the present study showed that midkine may 
be a diagnostic and prognostic biomarker for breast cancer.

\section{REFERENCES}

1. Sorkun HE, Akbulut M, Enli Y et al. (2016): Quantitative comparison of immuno-histochemical and PCR analysis of midkine expression in breast cancer types and serum midkine level. Turk J Med Sci., 46: 219-227.

2. Wang Y, Zhang Y, Pan C et al. (2015): Prediction of Poor Prognosis in Breast Cancer Patients Based on MicroRNA21 Expression: A Meta-Analysis. PLoS ONE, 10 (2): e0118647.

3. Zhang M (2016): Serum midkine expression in breast cancer patients and its clinical Significance. Journal of Hainan Medical University, 22 (2): 5-8.

4. Ibusuki M, Fujimori H, Yamamoto Y et al. (2009): Midkine in plasma as a novel breast cancer marker. Cancer Sci., 100: 1735-1739.

5. Guerrero RT, Novotny R, Wilkens LR et al. (2017): Risk factors for breast cancer in the breast cancer risk model study of Guam and Saipan. Cancer Epidemiology, 50: 221:233.

6. Jing X, Cui X, Liang $\mathrm{H}$ et al. (2017): Diagnostic accuracy of ELISA for detecting serum Midkine in cancer patients. PLOSONE, 12 (7): e0180511.

7. Haber G, Ahmed NU, Pekovic V (2012): Family History of Cancer and Its Association with Breast Cancer Risk Perception and Repeat Mammography.Am J Public Health, 102 (12): 2322-2329.

8. Macías-Gómez NM, Peralta-Leal V, Meza-Espinoza JP et al. (2015): Polymorphisms of the XRCC1 gene and breast cancer risk in the Mexican population. Fam Cancer, 14 (3): 349-354.

9. Brewer HR, Jones ME, Schoemaker MJ et al. (2018): Family history and risk of breast cancer: an analysis accounting for family structure.Breast Cancer Res Treat., 165 (1): 193-200.

10. Jiang AR, Gao CM, Ding JH et al. (2012): Abortions and breast cancer risk in premenopausal and postmenopausal women in Jiangsu Province of China. Asian Pac J Cancer Prev., 13: 33-35.
11. ELshmay WM (2016): The protective effect of longer duration of breastfeeding against pregnancy-associated triple negative breast cancer. Oncotarget,7 (33): 5394153950.

12. Khincha P, Best AF, Loud JT et al. (2018): Effect of breastfeeding on the risk of breast cancer in Li-Fraumeni syndrome. Journal of Clinical Oncology, 36 (15):1530.

13. Soroush A, Farshchian N, Komasi S et al. (2016): The Role of Oral Contraceptive Pills on Increased Risk of Breast Cancer in Iranian Populations: A Meta-analysis. J Cancer Prev., 21(4): 294-301.

14. Fauci A, Braunwald E, Kasper DL et al. (2008): A comparative study of pre- and post-menopausal breast cancer. Harrison's Principles of Internal Medicine, pp: 51622.

15. Kamińska M, Ciszewski T, Lopacka-Szatan $\mathrm{K}$ et al. (2015): Breast cancer risk factors. Prz Menopauzalny, 14 (3): 196-202.

16. Li F, Tian P, Zhang J et al. (2015): The clinical and prognostic significance of midkine in breast cancer patients. Tumor Biol., 36: 9789-9794.

17. Jones DR (2014): Measuring midkine: The utility of midkine as a biomarker in cancer and other diseases. British Journal of Pharmacology, 171: 2925-2939.

18. Kadomatsu K, Kishida S, Tsubota S (2013): The heparinbinding growth factor midkine: the biological activities and candidate receptors. J Biochem., 153 (6): 511-521.

19. Xu C, Zhu S, Wu M et al. (2014): Functional Receptors and Intracellular Signal Pathways of Midkine (MK) and Pleiotrophin (PTN). Biol Pharm Bull., 37 (4):511-520.

20. Nisman B, Maimon O, AllweisT et al. (2013): The prognostic significance of LIAISON(R) CA15-3 assay in primary breast cancer. Anticancer Res., 33 (1): 293-299.

21. Zhang D, Ding L, Li Y et al. (2017): Midkine derived from cancer associated fibroblasts promotes cisplatin-resistance via upregulation of the expression of lnc RNA ANRIL in tumor cells. Sci Rep., 7 (1): 16231-44.

22. Yamashita T, Shimada H, Tanaka S et al. (2016): Serum midkine as a biomarker for malignancy, prognosis, and chemosensitivity in head and neck squamous cell carcinoma.Cancer Medicine, 5 (3): 415-425.

23. Jono H, Ando Y (2010): Midkine: A Novel Prognostic Biomarker for Cancer. Cancers, 2: 624:641. 